\title{
Welche Rolle spielt die Ernährung bei Colitis ulcerosa?
}

Der Stellenwert der Ernährung in der Behandlung von $\mathrm{Pa}$ tienten mit Colitis ulcerosa wird in der von Bartels et al. vorgestellten Übersichtsarbeit [4] einerseits unter dem Gesichtspunkt einer Verbesserung der Ernährungssituation diskutiert, andererseits unter der (hypothetischen) Vorstellung, mit diätetischen Maßnahmen direkt in den zugrundeliegenden Entzündungsmechanismus eingreifen zu können. Trotz einer Fülle von Publikationen liegen bisher nur wenige gesicherte Studien zur Rolle der Ernährung in der Behandlung der Colitis ulcerosa vor. Für die meisten Patienten mit Colitis ulcerosa wird in Remissionsphasen eine nutritiv ausgewogene Diät mit minimalen Restriktionen empfohlen.

Eine supportive Ernährungstherapie ist indiziert bei $\mathrm{Pa}$ tienten mit schwer gestörter Darmfunktion oder Verdauungsstörungen sowie bei inadäquater Nahrungszufuhr. Bei schwer mangelernährten Patienten mit Colitis ulcerosa muß präoperativ eine Verbesserung des Ernährungsstatus erfolgen.

Untergewicht und Malnutrition sind aber bei der Colitis ulcerosa im Gegensatz z.B. zum M. Crohn relativ selten und im wesentlichen auf mangelnde Nahrungszufuhr im Rahmen krankheitsbedingter Beschwerden zurückzuführen. $\mathrm{Zu}$ den durch die Grunderkrankung bedingten Ernährungsproblemen kommen die Folgen der Steroidtherapie wie erhöhter nutritiver Bedarf; kataboler Eiweißstoffwechsel, reduzierte Wundheilung und vermehrte Infektanfälligkeit. Da die Colitis ulcerosa allenfalls im Rahmen einer Pankolitis das terminale Ileum erreicht (,,backwash-ileitis") kommt es nur selten zu einer relevanten Malabsorption [2]. Potentiell sind mit einem Ileumbefall ein Verlust der Gallensäuren- und der Vitamin- $B_{12}$-Resorption korreliert.

Inwieweit darüber hinaus der entzündliche Prozeß per se in der Akutphase der Colitis ulcerosa zu einem erhöhten Energieverbrauch führt, ist nicht gesichert [15].

C. Hanck $(\bowtie) \cdot$ M. V. Singer

IV. Medizinische Klinik (Schwerpunkt Gastroenterologie),

Klinikum Mannheim der Universität Heidelberg,

Theodor-Kutzer-Ufer 1, D-68167 Mannheim

\section{Supplementierende Rolle der Ernährung}

Allgemein wird davon ausgegangen, daß mangelernährte Patienten schlechtere Heilungschancen und ein größeres perioperatives Risiko aufweisen, wobei vor allem dem Infektionsrisiko Beachtung geschenkt wird [5].

Der Zusammenhang zwischen perioperativem Risiko und Ernährungsstatus konnte bei schwer malnutritierten Patienten mit Colitis ulcerosa erst vor kurzem in zwei großen prospektiven und kontrollierten Studien belegt werden. Unter total parenteraler Ernährung (TPN) bis zuweilen total enteraler Ernährung (TEN) reduziert sich parallel mit dem verbesserten Ernährungszustand (gemessen an Körpergewicht, Stickstoffbilanz und Serumeiweiß) die perioperative Komplikationsrate - und insbesondere die Zahl der postoperativen Infektionen $[18,25]$. Eine Verbesserung im Ernährungsstatus kann nachweislich ebenso effektiv mit enteraler (TEN - als polymere Diät verabreicht) wie mit parenteraler Ernährung (TPN) erreicht werden [8]. Dabei sollte die enterale Ernährung bevorzugt werden.

Hochmolekulare Diäten sind genauso effektiv wie Elementardiäten. Bei Patienten mit Laktoseintoleranz ist darauf zu achten, daß viele kommerziell erhältliche Diäten laktosehaltig sind. Aus folgenden Gründen sollte die TPN schwer kranken Patienten mit Kontraindikationen zur enteralen Ernährung und mit zuweilen ungenügender oraler Aufnahmefähigkeit vorbehalten bleiben:

1) Häufigere Komplikationen bei der parenteralen Ernährung als bei der enteralen Ernährung [9] (in einer Metanalyse erlitten signifikant weniger TEN- als TPNernährte Patienten septische Komplikationen [19]);

2) geringere Supplementierung von Eiweiß - die TEN mit polymerer Diät scheint der TPN bezüglich Eiweißsupplementierung ebenbürtig, wenn nicht überlegen -: so in der prospektiv randomisierten Studie von Gonzalez, in der signifikant höhere Serumalbuminwerte bei total enteraler (TEN) als bei total parenteraler Ernährung (TPN) gefunden wurden [9]; 
3) Mangelnde Verfügbarkeit einer umfassenden Supplementierung von Spurenelementen und Vitaminen - welche aus technischen Gründen nicht im gleichen Ausmaß wie in der enteralen Ernährung möglich ist;

4) Kostengründe;

5) Potentielle hypothetische Nachteile: Es wird diskutiert, daß die totale parenterale Ernährung die Dysfunktion der Darmbarriere verschlechtere, eine potentiell immunosuppressive Wirkung auf die Funktion der Neutrophilen und Lymphozyten habe und die Aktivität des Aminosäurenund Glukosetransportes in der Darmmukosa reduziere [1].

Zusammenfassend profitieren Patienten mit schwerer Malnutrition von einer präoperativen nutritiven Supplementierung, da hierdurch eine Reduktion größerer Komplikationen erzielt werden kann [18]. Eine längerdauernde parenterale Ernährung erscheint selten notwendig.

\section{Rolle der Vitamine und Spurenelemente in der Ernährung bei Colitis ulcerosa}

Weder das Vorkommen noch die pathogenetische Rolle eines Mangels an Vitaminen und Spurenelementen sind bei der Colitis ulcerosa bisher hinreichend geklärt.

Bei Patienten, die Salazosulfapyridin (SASP) über lange Zeit erhalten, kann wegen Resorptionshemmung der Folsäure eine Folatsubstitution indiziert sein. Es gibt Anhaltspunkte dafür, daß Folsäuremengel eine Rolle in der Entstehung der Kolonkarzinoms bei der Colitis ulcerosa spielen könnte [13, 14, 27]. Wie von Bartels et al. erwähnt, kann in bis zu 5\% der Fälle - und am ehesten im Rahmen einer „backwash-ileitis“ - ein Vitamin-B 12 -Mangel auftreten und eine Substitutionstherapie erforderlich sein [7]. Obwohl nur $15 \%$ der Patienten mit Colitis ulcerosa eine schwere hämorrhagische Diarrhö im Rahmen einer Pankolitis erleiden [8], haben $81 \%$ aller Patienten einen Eisenmangel, so daß eine Substitution erwogen werden sollte [7]. Patienten mit Steroidosteoporose können ggf. von einer Kalziumsupplementierung profitieren.

\section{Rolle der Ernährung als primäre Therapieform der Colitis ulcerosa}

Obwohl Bartels et al. erwähnen, daß ,einige Studien einen positiven Effekt im Hinblick auf die Indikation einer klinischen Remission oder eine Veränderung der Operationsindikation" belegt hätten, ist festzuhalten, daß keine randomisierte kontrollierte Studie je den wirklichen Nutzen irgendeines Types der primären Ernährungstherapie (eingesetzt statt konventioneller medikamentöser Therapie oder beim Versagen derselben), sei es mit elementarer Diät oder total parenteraler Ernährung (TPN) und „Darmruhigstellung", bewiesen hat [22].

So ließ sich das Konzept der „Darmruhigstellung“ bei (akuter) Colitis ulcerosa insbesondere in den prospektiv kontrollierten und randomisierten Studien von Dickinson et al. nicht bestätigen, in denen die Wirkung einer TPN respektive einer oralen Diät zusätzlich zur Steroidtherapie bei akuter Colitis ulcerosa untersucht wurde $[6,12,20]$. Damit bestätigten sich Ergebnisse früherer retrospektiver Studien $[3,21]$.

Zusammenfassend läßt sich somit sagen, daß die parenterale Ernährung nur wenig zum Management der akuten Colitis beiträgt - eine im Grunde alte Erkenntnis, die sich bereits in einer Review von Resultaten aus dem Jahr 1978 von Truelove findet [25].

\section{Die Rolle ungesättigter Fettsäuren in der Therapie der Colitis ulcerosa}

Patienten mit aktiver Colitis ulcerosa weisen erhöhte Leukotrienspiegel in der Rektummukosa auf, die mit der Krankheitsaktivität korrelieren [2]. Bei gesunden Frauen wurde beobachtet, daß eine orale Supplementierung mit $\omega_{3}$-Fettsäuren zu einer ,Downregulation“ proinflammatorischer Zytokine (IL-1 $\beta$, TNF, IL-6) führt [16].

Die Synthese der entzündungsmodulierenden Leukotriene kann durch die im Fischöl enthaltene Eicosapentaensäure gehemmt werden. In diesem Kontext wurden verschiedene klinische prospektiv-kontrollierte Studien durchgeführt in der Annahme, daß Fischölbestandteile potentiell auf den Entzündungsmechanismus inhibierend wirken könnten - ähnlich wie das in der Therapie der Colitis ulcerosa bewährte Sulfasalazin [22] und die 5-Aminosalicylsäure.

In den Ergebnissen der meisten dieser Studien wurde über klinische und histologische Verbesserungen unter $\omega_{3}$-Fettsäuren berichtet und ein verminderter Steroidbedarf beschrieben $[2,10,15,23]$. Im Gegensatz dazu konnte von Greenfield kein positiver Effekt auf die Krankheitsaktivität der Colitis ulcerosa bestätigt werden [10]. Die erwähnte Reduktion der Steroiddosis unter $\omega_{3}$-Fettsäuren war nicht immer signifikant geringer als in der Vergleichsgruppe unter Placebo [23]; in der Studie von Aslan wurde weder eine signifikante Besserung histologischer Parameter noch des Leukotriengehaltes der Mukosa bei Vergleich von peroraler Fischöl- bzw. Placeboapplikation gefunden [2]. In anderen Studien kam es zwar zu einem Abfall der Leukotriengehaltes in der Mukosa und zu einer Verbesserung des histologischen Index, klinisch konnte jedoch kein eindeutiger Effekt nachgewiesen werden.

Der Nutzen der $\omega_{3}$-Fettsäuren in der Therapie von Patienten mit Colitis ulcerosa scheint somit auf der Basis der vorliegenden klinischen Daten noch nicht definitiv gesichert zu sein. Hier sind weitere prospektive Untersuchungen notwendig.

\section{Literatur}

1. Ahrendt GM, Barbul A (1994) Nutrition and multiple organ dysfunction syndrome. Curr Opin Gastroenterol 10:203-209 
2. Aslan A et al (1992) Fish oil fatty acid supplementation in active ulcerative colitis. Am J Gastroenterology 87:432-437

3. Axelsson C, Jarnum S (1977) Asessment of the therapeutic value of an elemental diet in chronic inflammatory bowel disease. Scand J Gastroenterol 12:89-95

4. Bartels M, Nagel E, Pichlmayr R (1995) Welche Rolle spielt die Ernährung bei der Colitis ulcerosa. Langenbecks Arch Chir 380:4-11

5. Bodoky A, Neff U, Heberer M, Harder F (1993) Antibiotic prophylaxis with two doses of cephalosporin in patients managed with internal fixation for a fracture of the hip. J Bone Joint Surg Am 75:61-65

6. Dickinson RJ, Ashton MG, Axon ATR, Smith RC, Yeung CK, Hill GL (1980) Controlled trial of intravenous hyperalimentation and total bowel rest as an adjunct to the routine therapy of acute colitis. Gastroenterology 79:1199-1204

7. Dudrick S, Latifi R, Schrager R (1991) Nutritional Management of Inflammatory Bowel Disease. Current Strategies in Surgical Nutrition. Surg Clin North Am 71:609-623

8. Goebell H, Kölbel CBM, Zeitz M (1992) Colitis ulcerosa and M. Crohn. In: Goebell H (Hrsg) Gastroenterologie. Urban \& Schwarzenberg, München Wien Baltimore

9. Gonzalez-Huix F et al (1993) Enteral versus parenteral nutrition as adjunct therapy in acute ulcerative colitis. Am $\mathbf{J ~ G a}$ stroenterol 88:227-232

10. Greenfield SM, Green AT, Teare JP et al (1993) A randomized controlled study of evening primrose oil and fish oil in ulcerative colitis. Aliment Pharmacol Ther 7:159-166

11. Hawthorne $A B$ et al (1992) Treatment of ulcerative colitis with fish oil supplementation a prospective 12 month randomised controlled trial. Gut 33:922-928

12. McIntyre PB, Powell-Tuck J, Wood SR et al (1986) Controlled trial of bowel rest in the treatment of severe acute colitis. Gut 27: $481-485$

13. Lashner BA, Heidenreich PA, Su GL, Kane SV, Hanauer SB (1989) Effect of folate supplementation on the incidence of dysplasia and cancer in chronic ulcerative colitis: a case-control study. Gastroenterology 97:255-259

14. Lashner BA (1993) Red blood cell folate is associated with the development of dysplasia and cancer in ulcerative colitis. $J$ Cancer Res Clin Oncol 119:549-554

15. Lochs $\mathbf{H}$ (1992) Grundlagen der Ernährungstherapie bei chronisch entzündlicher Darmerkrankung. In: Gheorfhin TH, Kruis
W (Hrsg) Chronisch entzündliche Darmerkrankung. Aktuelle Therapiekonzepte. Symposium über chronisch entzündliche Darmerkrankungen. Wiesbaden

16. Lorenz $R$ et al (1989) Supplementation with $n-3$ fatty acids from fish oil in chronic inflammatory bowel disease - a randomised placebo-controlled double-blind cross-over trial. J Intern Med 225 [Suppl 1]:225-232

17. Meydani SN (1992) Modulation of Cytokine Production by Dietary Polyunsuturated Fatty Acids. Proc Soc Exp Biol Med 200: $189-293$

18. Meyenfeldt MF, Meijerink WJHJ, Rouflart MMJ, Buil-Maassen MTHJ, Soeters PB (1992) Perioperative nutritional support: a randomized clinical trial. Clin Nutr 11:180-186

19. Moore FA, Feliciano DV, Andrasy RI, Hope McArdle et al (1992) Early enteral feeding, compared with parenteral, reduces postoperative septic complications. Ann Surg 216: 172-183

20. Munkholm Larsen P, Rasmussen D, Ronn B, Munck O, Elmgreen J, Binder V (1989) Elemental diet: a therapeutic approach in chronic inflammatory bowel disease. J Intern Med 225: $325-331$

21. Rocchio MA, Cha CM, Haas KF, Randall HT (1974) Use of chemically defined diets in the management of patients with acute inflammatory bowel disease. Am J Surg 127:469-475

22. Silk DB (1992) Medical management of severe inflammatory disease of the rectum: nutritional aspects. Baillieres Clin Gastroenterol 6:27-41

23. Stenson WF et al (1982) Sulfasalzine inhibits the synthesis of chemotactic lipids by neutrophils. J Clin Invest 69:494-497

24. Stenson WF et al. (1992) Dietary supplementation with fish oil in ulcerative colitis. Ann Intern Med 116:609-614

25. The Veteran affairs total parenteral nutrition cooperative study group: Perioperative total parenteral nutrition in surgical patients (1991) N Engl J Med 325:525-532

26. Truelove SC, Willoughby CP, Lee EG, Kettlewell MGW (1978) Further experience in the treatment of severe attacks of ulcerative colitis. Lancet II: 1086-1088

27. Willadsen D, Schlepp GE, Tsuchida AM (1991) Dietary supplementation of folic acid is associated with lack of dysplasia in patients with chronic inflammatory bowel disease. Am J Gastroenterol 86: 1363

28. Williamson RC (1978) Intestinal adaption. N Engl J Med 298 $1393-1402$ 